\title{
Bronchiectasis complicating chronic lymphatic leukaemia with hypogammaglobulinaemia
}

\section{G K KNOWLES, R STANHOPE, AND M GREEN}

From the Chest Department, St Bartholomew's Hospital, London

Bronchiectasis may occur as a consequence of hypogammaglobulinaemia. ${ }^{1}$ It has also recently been reported in children with normal serum immunoglobulins who had acute lymphoblastic leukaemia. ${ }^{2}$ We report an adult with chronic lymphatic leukaemia complicated by depressed IgG and IgA levels in both serum and respiratory secretions who developed bronchiectasis and sinusitis while on chemotherapy. We can find no previous report of this association.

\section{Case report}

A 60-year-old man presented in June 1972 with malaise, generalised lymphadenopathy, and hepatosplenomegaly. He had been healthy previously with no history of childhood measles or whooping cough. He smoked six cigarettes per day, but had no cough or sputum. His chest was clear on examination and chest radiography was normal. His haemoglobin was $12 \cdot 0 \mathrm{~g} / \mathrm{dl}$, white cell count $180 \times 10^{9} / 1$ with $93 \%$ lymphocytes, $3 \%$ neutrophils, and $2 \%$ monocytes, and platelet count $70 \times 10^{9} / 1$. Bone marrow showed a hypocellular specimen composed mainly of lymphocytes with scanty megakaryocytes. Treatment was started with chlorambucil $2 \mathrm{mg}$ daily increasing to $4 \mathrm{mg}$ daily.

In July 1973 he was admitted with fever and productive cough after a sore throat. White cell count was $3.6 \times 10^{9} / 1$ with $75 \%$ lymphocytes, $25 \%$ neutrophils, and platelet count $60 \times 10^{9} / 1$. His chest radiograph was normal. All cultures proved negative and his fever settled spontaneously. Chlorambucil was withdrawn temporarily with subsequent improvement in white cell count and prednisolone $15 \mathrm{mg}$ daily was started because of persisting thrombocytopenia. Hypogammaglobulinaemia was noted on protein electrophoresis.

During 1974 his cough worsened with up to one egg-cupful of purulent sputum daily and he developed nasal catarrh. His chest radiograph now showed bilateral lower zone shadowing. Ear, nose, and throat examination was normal. Over the next four years he was admitted on five occasions with fever, breathlessness, and increase in sputum, but no haemoptysis. On examination there were persistent bilateral basal crackles, but no clubbing. The chest radiographic abnormalities remained unchanged.

Address for reprints: Dr $\mathbf{M}$ Green, Chest Department, St Bartholomew's Hospital, West Smithfield, London EC1A 7BE.
Serum immunoglobulins were IgG $3 \mathrm{IU} / \mathrm{ml}$ (normal range 57-172 $\mathrm{IU} / \mathrm{ml}$ ), IgA $17 \mathrm{IU} / \mathrm{ml}$ (normal range 74-268 IU $/ \mathrm{ml}$ ), and IgM $140 \mathrm{IU} / \mathrm{ml}$ (normal range 58-197 IU/ml). Sputum cultures were negative for bacterial pathogens including acid-fast bacilli, and for fungi. No malignant cells were seen. Heaf test was negative. Serological tests for viruses, Mycoplasma pneumoniae, fungi, and Pneumocystis carinii were also negative. Rigid bronchoscopy was normal. Percutaneous drill lung biopsy of the right lower lobe revealed minor inflammatory changes, but no leukaemic infiltration or pulmonary fibrosis. The patient was treated with courses of antibiotics and postural drainage, and received three doses of $1500 \mathrm{mgs}$ of gammaglobulin intramuscularly at two weekly intervals with slight improvement. Prednisolone was reduced to $10 \mathrm{mg}$ daily and he continued to receive intermittent courses of chlorambucil.

A bronchogram in February 1978 showed extensive bronchiectasis in both lower lobes, right middle lobe, and lingula (figure). Serum, sputum, and nasal secretion immunoglobulin levels were measured using Hoechst Tri-partigen and LC partigen plates and showed reduced IgG and IgA concentrations in all specimens (table). Lung function tests showed a moderate obstructive defect (forced expiratory volume in one second 1.251 -predicted 2.45-3.31 1 ; forced vital capacity $2.88 \quad 1$-predicted $3.33-4.5 \quad 1$ ) with reduced diffusing capacity for carbon monoxide, 3.99 mmol $\min ^{-1} \mathrm{kPa}^{-1}$. Sinus radiographs showed mucosal thickening in the right maxillary antrum with later bilateral antral opacification. After treatment with further courses of antibiotics, postural drainage, bronchodilators, Iliadin nose drops, and steam inhalations his chest condition improved.

In May 1978 the patient was finally admitted to another hospital suffering from septicaemia and died. A necropsy was not performed.

Table Serum, sputum, and nasal secretion immunoglobulin levels $(I \mathrm{U} / \mathrm{ml})$

\begin{tabular}{lcll}
\hline & Serum & Sputum & Nasal secretion \\
\hline IgG & $15(57-172)^{*}$ & $1(12 \pm 9) \dagger$ & $1(6 \pm 4) \ddagger$ \\
IgA & $6(74-268)$ & $9(55 \pm 39)$ & $5(26 \pm 14)$ \\
IgM & $267(58-197)$ & Not done & Not done \\
\hline
\end{tabular}

* Normal range

+ Mean $\pm 2 S D$ of 11 patients with bronchiectasis and normal serum IgG and IgA levels

$\ddagger$ Mean \pm 2 SD of 30 normal subjects 


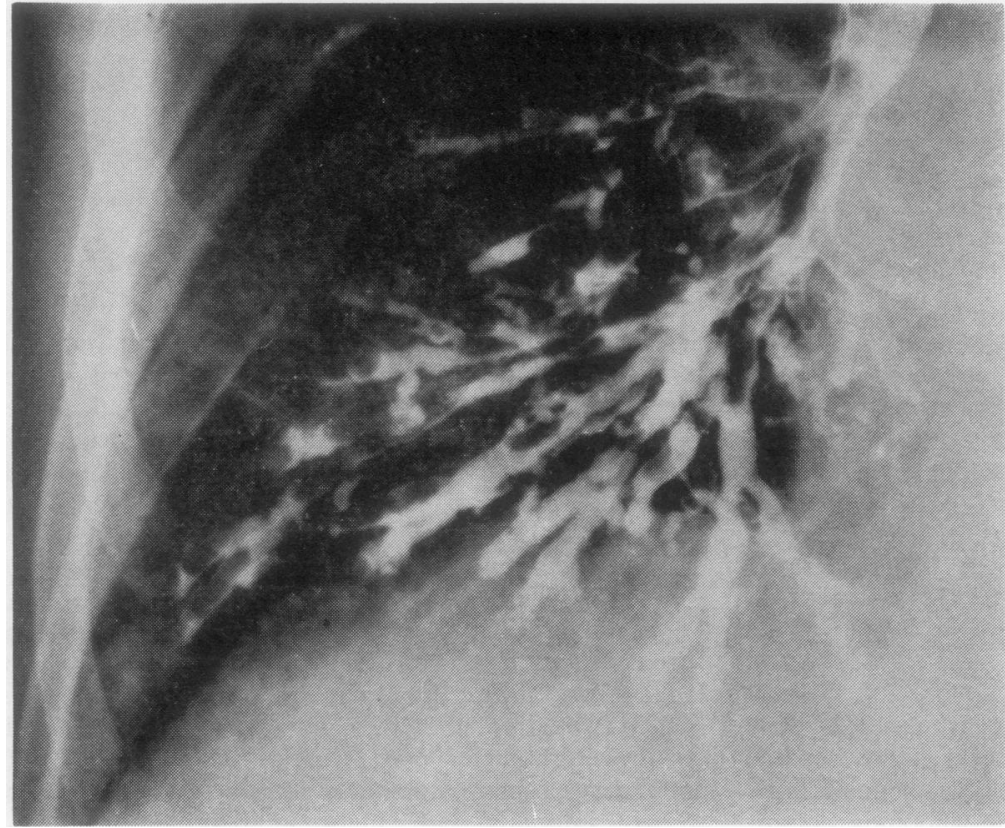

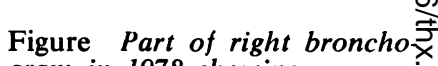
gram in 1978 showing bronchiectasis in right middle $\mathcal{W}$ and lower lobes

\section{Discussion}

This patient with chronic lymphatic leukaemia and hypogammaglobulinaemia developed extensive bronchiectasis and maxillary sinusitis. Bronchiectasis is well described in childhood as a complication of hypogammaglobulinaemia and other primary immunodeficiency disorders ${ }^{13}$ and has recently been reported in children with normal serum immunoglobulin levels who had acute lymphoblastic leukaemia. $^{2}$ Possible predisposing factors in these leukaemic patients included neutropenia and recurrent upper respiratory tract infections. In adults bronchiectasis is common in idiopathic late-onset immunoglobulin deficiency, ${ }^{4}$ but as far as we are aware has not been reported in association with leukaemia. Since hypogammaglobulinaemia may occur in up to $67 \%$ of patients with chronic lymphatic leukaemia, ${ }^{5}$ bronchiectasis could complicate this disease more frequently than hitherto suspected. In our patient it seems likely that the depressed levels of $\operatorname{IgG}$ and $\operatorname{IgA}$ in serum and respiratory secretions predisposed to the development of bronchiectasis and sinusitis. It is probable however that other factors such as neutropenia, and impaired cellular immunity, related either to the underlying leukaemia or to its treatment, may have played important roles. It is unlikely that sinus infection contributed to the bronchiectasis since radiographic evidence of bilateral maxillary sinusitis was only obtained after the bronchiectasis had been clinically established for four years.

Bronchiectasis and sinusitis should be considered as potential hazards in patients with leukaemian particularly if they have associated hypogamma-D globulinaemia. An awareness of these possible complications with early diagnosis and active treat-o ment of acute respiratory infection may help prevent ${ }^{\Im}$ chronic infection. Bronchography can assist in differentiating bronchiectasis from other respiratory problems in these patients and may obviate the need for more invasive diagnostic techniques. Our patiento improved on gammaglobulin therapy, regular postura drainage, and intermittent antibiotics. Such treatmento should be implemented as soon as bronchiectasis is 3 . confirmed.

We thank Dr AE Dormer and Dr RS Francis for permission to report details of this patient.

\section{References}

1 Good RA, Mazzitello WF. Chest disease in ${ }^{\circ}$ patients with agammaglobulinaemia. Dis Ches N 1956; 29:9-35.

2 Kearney PJ, Kershaw CR, Stevenson PA. Bron- chiectasis in acute leukaemia. Br Med $J 1977$. 2:857-9.

3 Lawler GJ, Ammann AJ, Wright WC, Lके Franchi SH, Bilstrom D, Stiehm ER. The synes drome of cellular immunodeficiency with immunoglobulins. J Pediatr 1974; 84:183-92.

4 Dukes RJ, Rosenow EC, Hermans P. Pulmonary manifestations of hypogammaglobulinaemia Thorax 1978; 33:603-7.

5 Hamilton Fairley G, Bodley Scott R. Hypo gammaglobulinaemia and chronic lymphatic leukaemia. Br Med J 1961; 2:920-4. 